\title{
A Cost Effective Smart Antenna for Mobile Broadband Systems
}

\author{
S.Ravishankar, PhD. \\ Professor, ECE Department \\ RV College of Engineering \\ Bangaore(India)
}

\author{
Prakash Biswagar \\ Associate Professor, ECE Department \\ $\mathrm{RV}$ College of Engineering \\ Bangaore(India)
}

\begin{abstract}
A cost effective implementation of a 'Smart Antenna' is limited due to the number and high cost of RF array elements, RF distribution as well as phasing elements. Shaped Dielectric Lenses perform the task of collimation, to obtain multiple beams in a fixed set of directions. Lenses are inherently broadband, lower in cost, easy to fabricate, have lower dimensional tolerances, and act as a radome for the electrically small primary radiators that are embedded inside or placed behind the lens.
\end{abstract}

Key Words: MBS, Collimation, Dielectric Lens, Radome, Primary Radiators

\section{Introduction}

The foreseeable fast growing market of the high quality multimedia applications and the internet, with ever increasing bandwidth requirements, is expected to strive, in the near future, for a full extension to the mobile users of the capacity available in the fixed Broadband Integrated Services Digital Network (B-ISDN) [1,2]. The cellular Mobile Broadband Systems (MBS) concept was introduced with this motivation.

It is intended to transport in a transparent way, Asynchronous Transfer Mode (ATM) cells over the air interface at the data rates up to $155 \mathrm{Mbps}$, withstanding mobile velocities up to $100 \mathrm{Kms}$ per hour, while maintaining the quality of service of fixed networks. Provisional sub bands were allocated for these systems at $39.5-40.5,42.5-43.5$ and $62-63,65-66 \mathrm{GHz}$.

The high attenuation in the millimeter wave bands makes frequency reuse very efficient. But at the same time, the use of millimeter waves pose added challenges to the design of broadband high mobility communication systems i.e. low power available from solid state devices, severe fading due to multipath effects and higher diffraction losses, which makes blocking by obstacles very critical.

\section{MBS and the Antenna Requirements}

MBS poses new challenges in addition to those dealt with by the lower rate mobile systems. Figure 1 below compares the different mobile systems in terms of mobility and data rates.

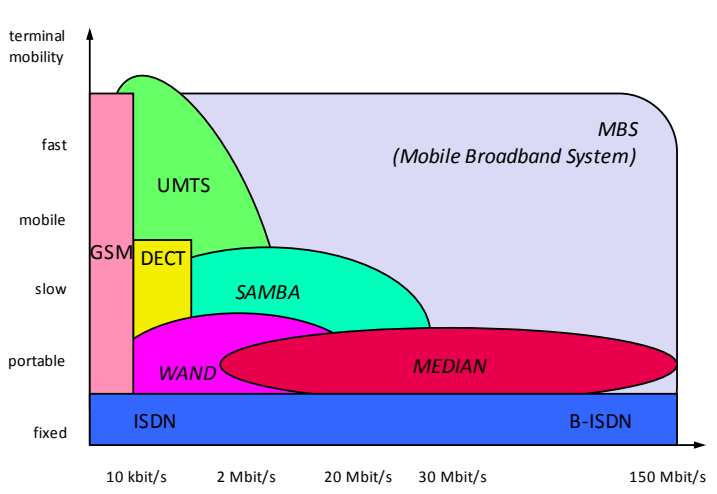

Fig. 1 Mobile Network Standards

On one hand, the high data rates envisaged for MBS requires operation at much higher radio frequencies, like the $60 \mathrm{GHz}$ bands, on the other hand, from a network point of view, the user applications using the higher available capacity might also place stringent performance requirements on the network. Figure 2 below shows a typical configuration of a shaped lens antenna system.
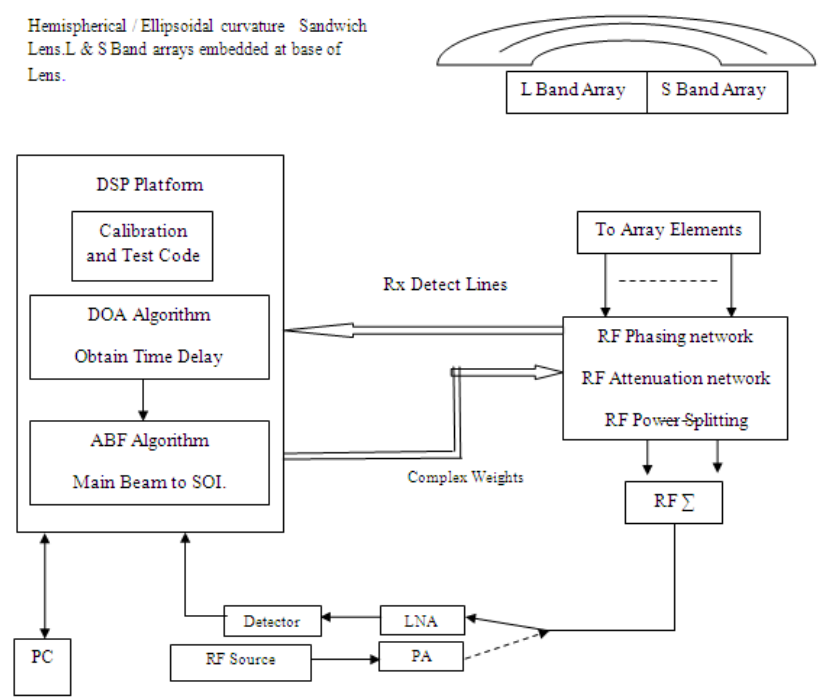

Fig. 2 A simplified system Architecture of Array- Lens antenna 
To achieve a high rate goal in such applications, 'Smart Antennas' were proposed at base stations for the newly opened bands in $5,11 \mathrm{GHz} \& \mathrm{~mm}$ wave sub bands for MBS. However a cost effective implementation of a smart antenna is limited due to high cost of RF array elements, RF distribution and phasing. Then the other alternatives are the fixed shaped beam antennas which offer an excellent compromise between cost and system performance especially in an urban scenario where dense traffic occurs in specified directions only.

Antenna beam shaping is a simple strategy to act on the above said parameters in a controlled way. No added complexity results to the system in the case of fixed shaped-beam antennas; on the contrary, with appropriate beam shaping, propagation of channel impairments can be mitigated to the point that equalizer complexity can be significantly reduced.

The mobile systems require carefully designed antennas at both the base station and the user or Mobile Terminals (MT) for efficient operation. It is essential to carefully control radiation patterns to target coverage to desired coverage areas while minimizing interference outside [2,3].The antenna structure should minimize interactions with its surroundings, such as supporting structures and the human body, while maximizing the efficiency for radiation and reception.

Given the low gain of the antennas, it is important to achieve a high translation of input RF power into radiation over the whole range of conditions of use. Generally, the MT antennas must be as small as possible, consistent with meeting the performance requirements. Increasingly, the ability to adapt the shape to fit into casing acceptable to a consumer product is important.

\section{Shaped Dielectric Lens Antennas}

There is no best antenna solution to cope with all the above said conflicting requirements. The choice between the available solutions must be made according to each service specific needs. Shaped dielectric lenses are one solution that is attractive for its simplicity, flexibility and effectiveness to comply with different specifications for wireless millimeter wave systems.

Shaped Lens Antennas are cost effective for microwave \& millimeter wave bands and offer a good compromise between simplicity and system performance.

- Dielectric lenses can be used for beam shaping and providing a collimation solution.

- Lenses are quite inexpensive, since they can be moulded and have a good fabrication tolerance.

- The feed is located behind the radiating surface and can be easily embedded in the lens to favour wide angle radiation required in most situations.

- Lenses can easily accommodate large bandwidths required by high data rate systems.

- The main drawback is internal reflections.

- Internal dissipation losses are negligible with dielectric materials available having low loss tangents through $\mathrm{mm}$ wave frequencies.

- Lens solutions are mechanically more robust and also double up as protective radome for primary radiators.
Figure 3 below shows a dielectric lens antenna configuration suitable for wireless applications.

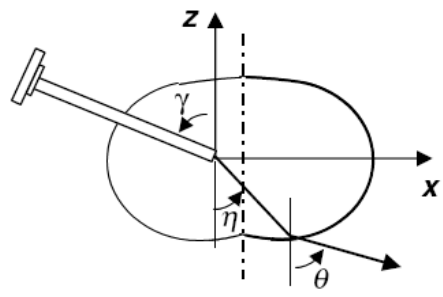

\section{Fig. 3 Geometry for axial-symmetrical lens with off-axis} excitation.

Generally, it is a homogeneous dielectric body with an arbitrary three dimensional shaped surface, which is designed to transform the feed radiation pattern into the desired output pattern. Possible feeds may be the aperture of rectangular or circular waveguides, coaxial feeds or printed elements either with linear or circular polarization.

Unlike conventional lens configuration, the feed is embedded in the lens material. This has important advantages over the usual configuration where the feed is located outside the lens body as follows.

- Broad-beam feeds can be used to illuminate the widest possible area of the lens surface with less spillover, favouring lens radiation for wide $\theta$ angles using moderate dielectric permittivity values.

- It is easier to promote an effective power transfer from the embedded feed into the lens.

- The total antenna depth is smaller when compared with alternative configurations where the feed is located at some distance away from the lens.

In the configuration shown above, only one of the lens surfaces intercepts the main beam of the feed, to shape it into a prescribed target radiation pattern; this is a single surface lens. It means that only one degree of freedom is available for pattern synthesis. Because amplitude shaping of the output beam is the only requirement for most of the wireless millimeter wave applications, a single degree of freedom is enough for the lens design.

Although the dielectric lens may have an arbitrary three dimensional shape, the axis - symmetrical case is the most common. Still various useful forms of asymmetrical cell shapes can be obtained simply by appropriate off-axis positioning of the feed, inside the axial-symmetrical lens.

\section{Dielectric Materials}

For the applications in the frequency range of $50-60 \mathrm{GHz}$, the $\epsilon_{\mathrm{r}}$ values typically range from 2 to $3[7,11 \& 12]$.This represents a compromise between the ability to obtain wide angle lens refraction and the requirement for the lowest internal reflection losses. Because of large lens dimensions in terms of wavelength, dissipation losses are also an important aspect to mind. Total dissipation losses depend on lens dimensions, shape and illumination functions.

The design procedure comprises two steps. In the first one, the lens profile is obtained from the geometric optics formulation, therefore assuming that lens dimensions are very large in terms of wavelength. 
Physical optics is used in the second step to obtain the corresponding output radiation pattern, taking diffraction effects into account. This pattern may be slightly different from the geometric optics prediction depending on the lens size. A slight lens profile correction may be introduced to bring the output radiation pattern closer to the target.

\section{Primary Radiators}

The success of the lens design to obtain a prescribed radiation pattern depends partly on the adequate choice of the feed. Besides the feed, direct implication on lens output pattern and polarization characteristics, the feed beam shape may affect the lens depth, the amount of internal reflection and the quality of the approximation to the target pattern for a given lens size. Some optimization of the feed may be useful to enhance these parameters. Feed characteristics that are relevant for the lens designs are

- $\quad$ Pattern shape (beam width, circular symmetry)

- Polarization

- Phase center location with respect to feed aperture

- Matching at the lens input port

It should be noted that for the present antenna configuration the preceding characteristics must be known when the feed is immersed in the lens material.

The lens materials could be Teflon, Polystyrene foam, Perspex, Polyurethane foam or even foam with suspended dielectric particle. A dielectric lens could also be used to enhance the gain of an existing array over a limited sector due to collimation effects.

A primary radiator and dielectric lens combination is cost effective from a number of viewpoints.

- Small sizes of primary radiator arrays as compared to much larger $(8 \times 8,16 \times 16)$ array sizes for a fully adaptive array.

- Mutual coupling effects are far less and more easily handled by positioning array elements.

- Manufacturing tolerances are more relaxed by using dielectric lenses.

- Multiple band operation is easily achieved by multiple small primary radiators but same lens, since dielectric lenses are inherently broadband.

- Multiple beam operation is easily achieved by a) multiple primary radiators b) generating multiple beams from a radiator array, with a higher directivity being provided by lens collimation.

- A sandwich of dielectric materials could be used to control internal reflections and achieve better match.

- Dielectric lens provides mechanical rigidity and functions as a radome for the underlying primary radiators.

- Since there are fewer elements in the primary radiator, the algorithms for $\mathrm{DOA}$ and $\mathrm{ABF}$ would need much less signal processing, thereby affording resizable real time operation and cost.
The dielectric lens shape and the materials are usually chosen to satisfy

- Structural and environmental requirements.

- A single homogeneous shell or several distinct layers.

- With the frequencies specified, electromagnetic design determines the thickness of various layers, the materials (subject to environment requirements such as elevated temperatures produced during high speed flight) and variations in thickness to obtain values of transmittance, bore sight error and side lobe levels.

\section{Dielectric Lens Design Considerations}

A dielectric lens is a window of a definite shape and size used to protect an antenna, which is radiating or receiving electromagnetic energy or both, in the frequency range from $\mathrm{dc}$ to $1000 \mathrm{GHz}$ and is designed to preserve the radiation or receiving function or both of the device with minimum aberration and maximum efficiency.

- Electrical requirements - This requirement consist of any design problems with a window transparent to EM energy of the desired frequency range and intensity.

- Mechanical requirements - Design of structure and all other requirements associated with the ability of the dielectric lens to withstand desired environmental conditions (wind loads, inertial loads, high temperature due to aerodynamic heating, low temperatures, moisture resistance, rain erosion resistance, shock and vibration, dust erosion and impact, icing lightning strikes etc.,). Electrical design may not be changed without changing mechanical design and vice-versa.

\section{Fabrication of Reference Antenna}

The spherical dielectric lens diameter depends on the size of the primary radiator which is placed behind the dielectric lens and the depth determines the collimated beam. The spherical lens was designed keeping in mind two rectangular waveguides, with a separation greater than the operating wavelength, as the primary radiator. The diameter for the lens was selected as $120 \mathrm{~mm}$, which includes a tolerance of half the operating wavelength. The spherical dielectric lens antenna was built to operate in $\mathrm{X}$ - band to facilitate experimentation in the laboratory. While selecting material for lens we made a survey on available dielectric materials and their loss tangent. Teflon material was the obvious choice, as a dielectric to fabricate the lens, because of low loss tangent (0.00037) and easy to fabricate, easily capable of providing good collimation with less loss. The material was available as a rod of diameter $120 \mathrm{~mm}$ and length of $1 \mathrm{ft}$.

The fabrication was done using a turning machine as shown below in figure 4 under the specified tolerance level. The required finishing was provided by surface grinding. The resulting spherical lens of diameter $120 \mathrm{~mm}$ is as in figure 5 which exactly matched the desired profile. 


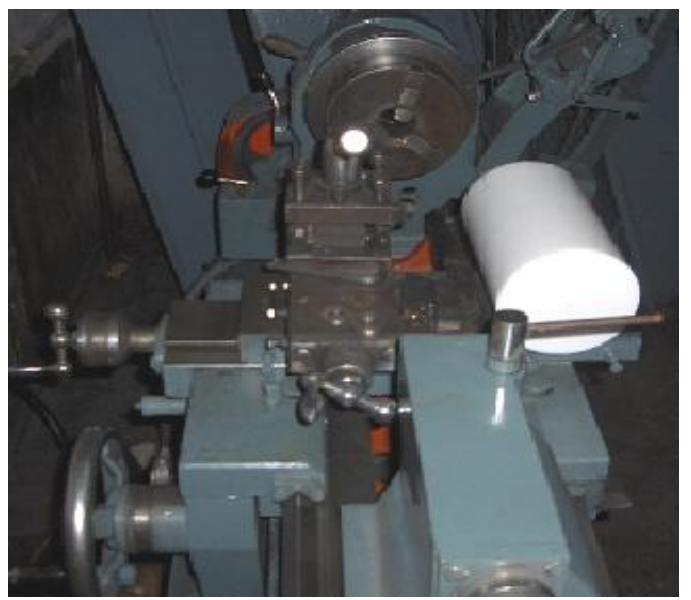

Fig. 4 Teflon rod on the turning machine

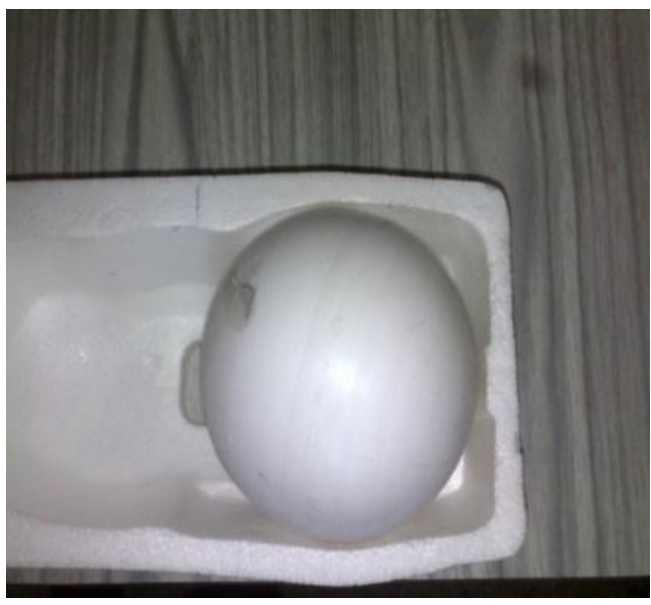

Fig. 5 Lens of diameter $12 \mathrm{cms}$

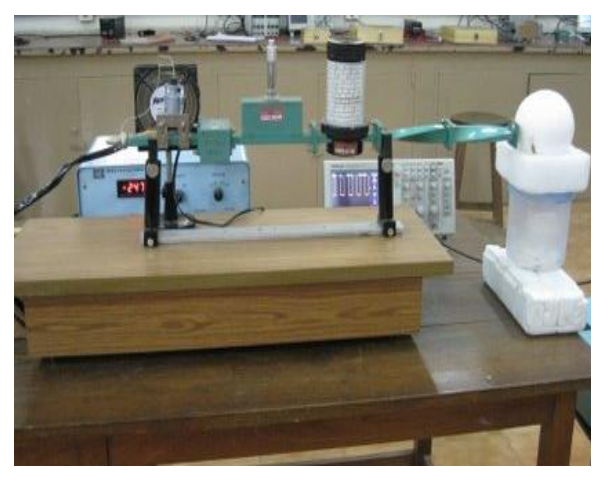

Fig. 6 Lens in front of a Rectangular Waveguide Anerture

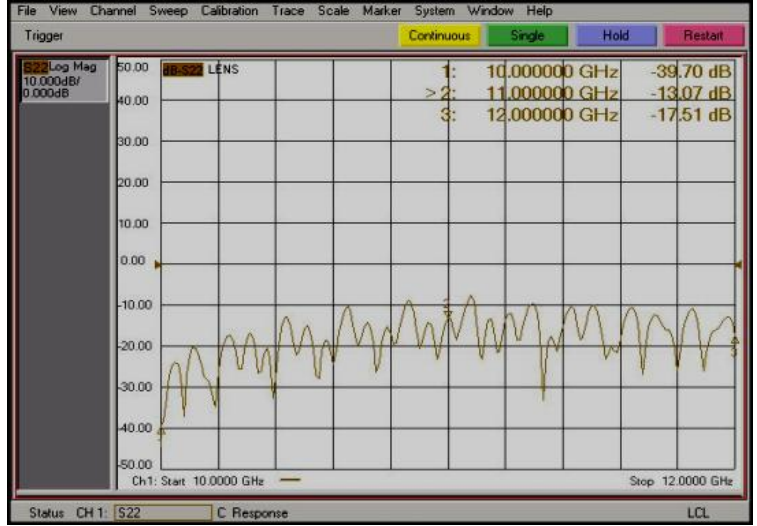

Fig. 7 Return losses for the spherical lens

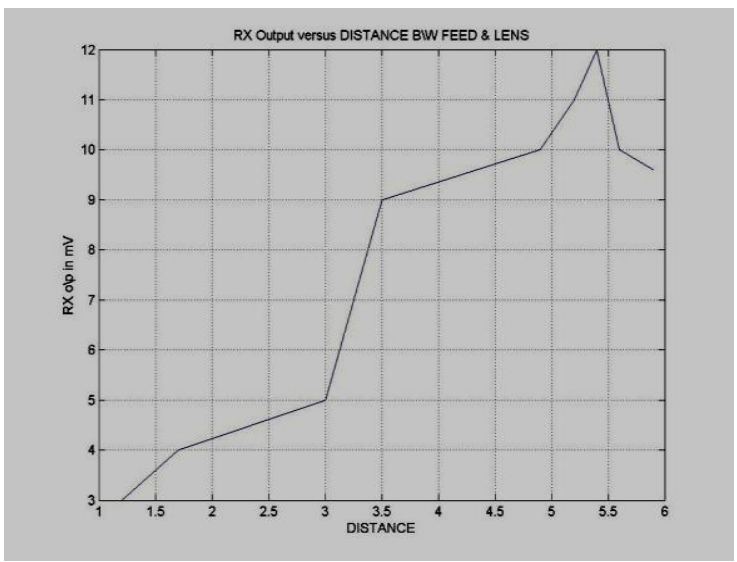

Fig. 8 Plot of VSWR versus distance between aperture and lens.

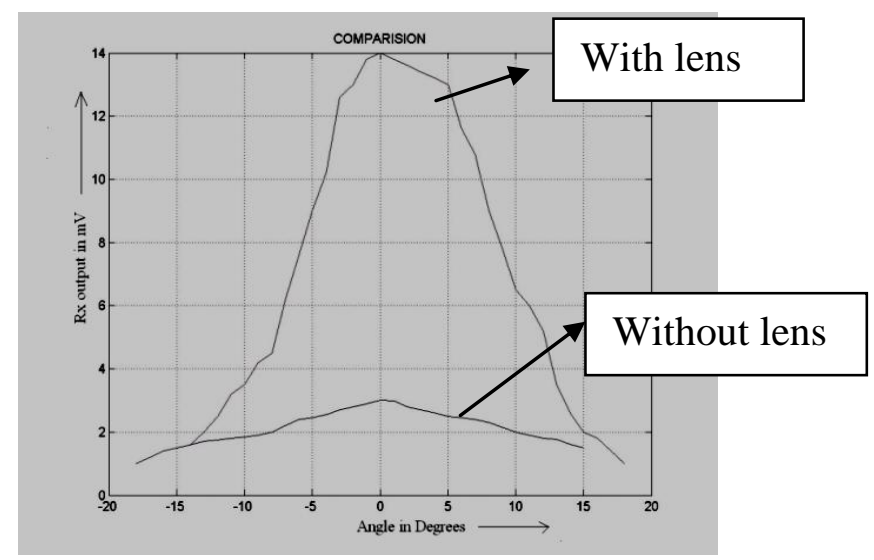

Fig.9 Comparison of experimental results with and without lens(E-field) 


\section{Lab Testing}

The lab testing was performed with the primary motive of proof of collimation and completed in three phases.

1. Obtain rectangular waveguide field pattern without using lens.

2. Determination of minimum VSWR in the rectangular waveguide in the presence of the lens.

3. Obtain the rectangular waveguide field pattern with the lens positioned in front of the aperture for the minimum VSWR case

The spherical lens is embedded on a pedestal of foam (dielectric constant of 1.0008) as shown in figure 6 above.

In order to obtain best possible radiation efficiency, it is needed to minimize the VSWR looking at the feed input. A separate experiment was done to determine the optimum distance between the lens and the primary radiator, where a return loss was better than $18 \mathrm{db}$. Figure 7 above shows the return loss plot for the spherical lens. The values obtained at different distances are plotted as shown above in figure 8 . From the graph we observe that the optimum distance at minimum VSWR is $5.4 \mathrm{cms}$. The lens holder is placed in front of the rectangular waveguide aperture antenna at the minimum VSWR position and field strength was measured in the principal planes.

The E-plane field pattern results with Lens are plotted in figure 9 above. For comparison purpose the field pattern without lens is also given in the same graph. The pattern was obtained taking only main beam into consideration.

From the plot it is clear that with the lens we get better collimation and the HPBW was reduced. The effect of collimation was clearly evident with a substantial reduction in HPBW to $21^{0}$ (with lens) from nearly $68^{\circ}$ (without lens) accompanied by an increase in directive gain of about $11 \mathrm{~dB}$.

\section{Field Testing in an Anechoic Chamber}

To study the effects of lens collimation, a Microstrip Patch antenna to work in $2.7 \mathrm{GHz}$ was fabricated on a RF grade substrate RT Duroid $5880\left(\varepsilon_{\mathrm{r}}=2.2\right)$ which is shown below in figure 10 .

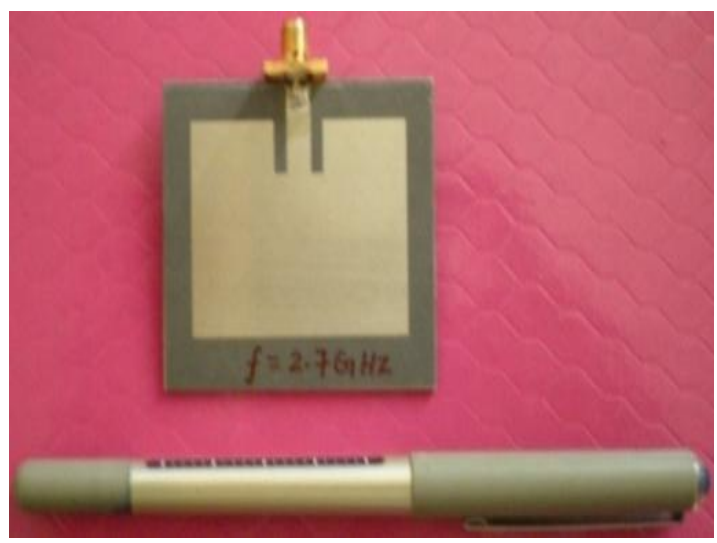

Fig. 10 The Micro strip Patch Antenna used as a Primary radiator.

The available standard formulae for calculation of the micro strip antenna dimensions were used [5, 12]. The patch antenna design steps are as below.

\section{Design Specifications}

2. Calculation of the antenna dimensions using empirical formulas

3. Simulation and optimization of the antenna structure using commercially available software

4. Check the antenna parameters like VSWR, impedance, return loss, gain etc.

5. Tune the parameters till the they are acceptable.

6. Take the photo film of the simulated file and fabricate the antenna using the substrate.

7. Test the antenna for its pattern and other parameters.

The rectangular patch was made to radiate at a frequency of $2.5 \mathrm{GHz}$, in front of the reference Teflon sphere of diameter $12 \mathrm{cms}$ in an anechoic chamber as shown in figure 11 below. The distance of separation between the patch and center of the dielectric lens was kept at $5 \mathrm{~cm}$, where the reflection losses were better than $-20 \mathrm{~dB}$. To perform the measurement, the dielectric sphere was kept on a supporting stand made of polystyrene foam with $\varepsilon_{\mathrm{d}}=1.08$ practically same as free space, so that the radiation pattern of the aperture-lens combination does not get perturbed. Figure 12 below shows the return losses of the patch

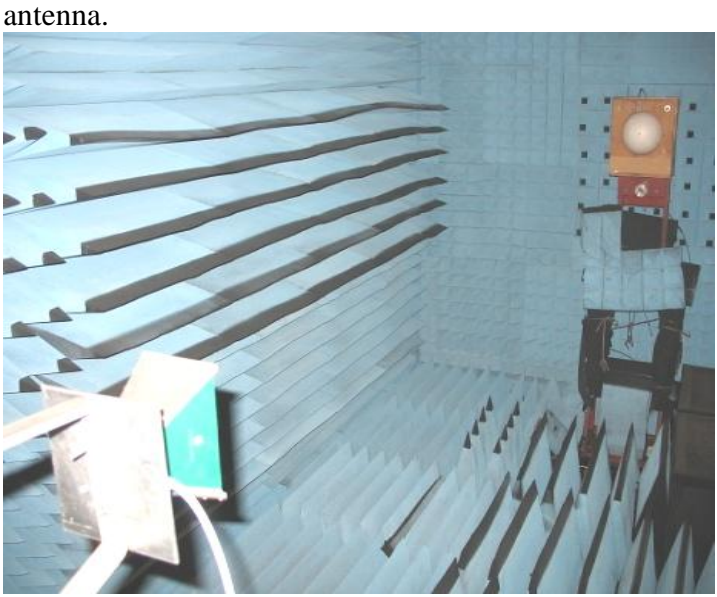

Fig. 11 Lens Setup in an Anechoic chamber

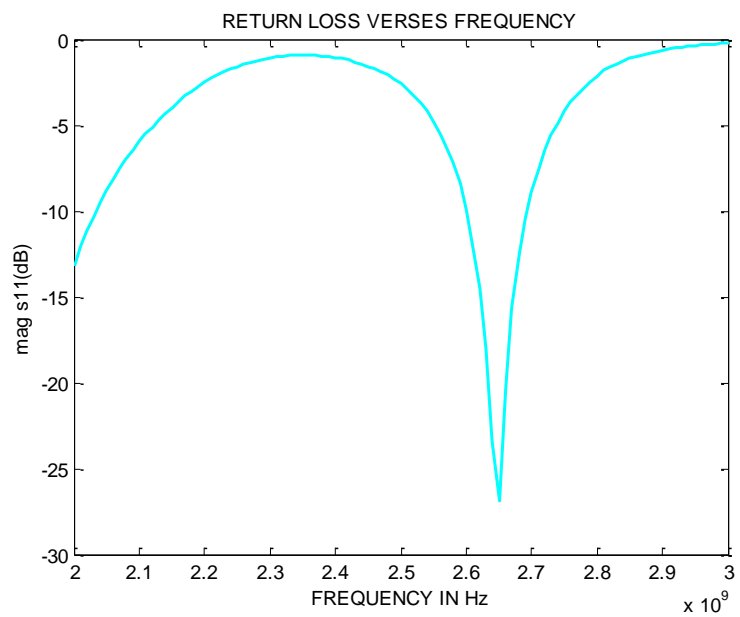

Fig.12 Return Loss of the Patch Antenna 
The measured field patterns are shown in figures 13 and 14 below. The effect of collimation was easily observed both

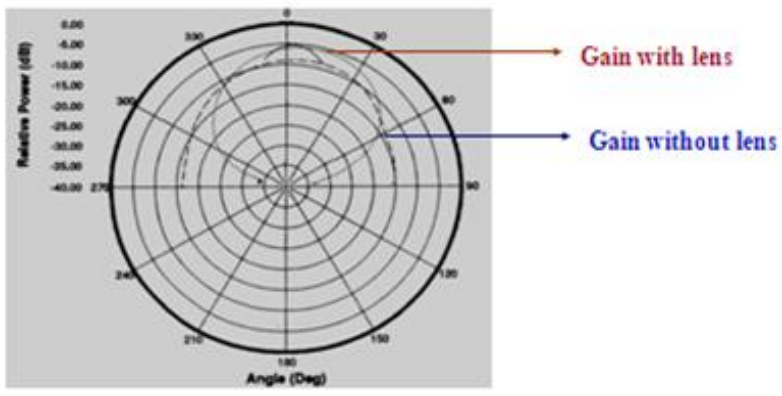

Fig. 13 E-Plane Pattern Patch-Lens combine (Small dashed line is that of the standard gain horn) in figures and summarized in Table1.

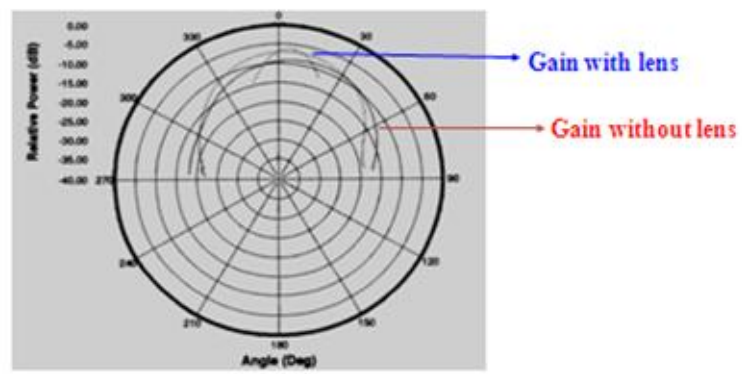

Fig.14 H-Plane Pattern Patch-Lens combine (Small solid line is that of the standard gain horn)

\begin{tabular}{|c|c|c|}
\hline & Without Lens & With Lens \\
\hline Gain (SGH = 10 dbi) & $6.3 \mathrm{dbi}$ & $9.4 \mathrm{dbi}$ \\
\hline $\begin{array}{c}\text { Beamwidth H - } \\
\text { Plane }\end{array}$ & 102 degrees & 80 degrees \\
\hline
\end{tabular}

Table1: Comparison of gain with and without lens antenna

\section{Conclusion}

Thus a dielectric lens antenna with radiating elements embedded inside or placed behind it promises as an efficient, affordable and cost effective antenna for MBS and now the search should be for optimal geometrical configurations for the lens and the range of applications, where these could be exploited.

\section{References}

[1] Carlos A Fernandes, "Shaped dielectric lenses for Wireless Millimeter-Wave Communications,"IEEE Antennas and Propagation Magazine, vol. 41, No 5, pp 141-150, October 1999.

[2] Leandro Fernandes, "Developing a system concept \& Technologies for Mobile Broadband Communications" IEEE Personal Communications Magazine, pp 54-59, Feb 1995.

[3] Carlos A Fernandes, Jose G Fernandes, "Performance of Lens Antennas in Wireless Indoor Millimeter Wave Applications," IEEE Transactions on Microwave theory and Techniques, vol47, No 6, pp732-736, June 1999.

[4] S.Ravishankar and M.S. Narasimhan, "Multiple Scattering of EM Waves by Dielectric Spheres located in the near field of a source of Radiation,"IEEE Transactions on Antennas and Propagation, vol AP-35, No 4,pp 399-405, April 1987.

[5] Constantine A Balanis, Antenna Theory - Analysis and Design, 2nd Edn, Singapore: John Wiley and Sons: Chapter 12, 2002.
[6] Constantine A. Balanis,Advanced Engineering Electromagnetics, John Wiley \& Sons, New Jersey, 1989.

[7] Carlos A.Fernandes et al., Handbook of Antennas in Wireless Communications, CRC Press, 2002.

[8] John Thornton, "Properties of spherical lens antenna for high altitude platform Communications," http: //www.ece.unm.edu/summa/notes/ssn/note382.pdf.

[9] Tin Komljenovic, "Lens Antenna - Analysis and Synthesis at $\mathrm{mm}$ waves,"http://www.fer.hr/download/repository/kvalifikas cijski rad komljenvic.pdf.

[10] Reuven Shavit, "Dielectric spherical lens antenna for mm-wave communications," Microwave and Optical Technology Letters, " vol.39, No.1, Oct.5, 2003.

[11] Simon R. Saunders et all, Antennas and Propagation for Wireless Communication Systems, John Wiley \&Sons, 2007

[12] Constantine A .Balanis,edt, Moderen Antenna Handbok,John Wiley \& Sons, 2007

\section{Acknowledgement}

The authors wish to thank the Naval Research Board, Defense Research and Development Organization, Govt of India and R $\mathrm{V}$ college of Engineering, Bangalore - 560059, India, for their support and assistance in this project. 